\title{
Sensorimotor Processing in the Basal Ganglia Leads to Transient Beta Oscillations during Behavior
}

\author{
(DAmin Mirzaei, ${ }^{1,2} \oplus^{\oplus}$ Arvind Kumar, ${ }^{3,4}$ Daniel Leventhal, ${ }^{5}$ CNicolas Mallet, ${ }^{6}{ }^{\oplus}$ Ad Aertsen, ${ }^{2,4}$ Joshua Berke, ${ }^{7}$ \\ and ${ }^{-}$Robert Schmidt ${ }^{8}$ \\ ${ }^{1}$ BrainLinks-BrainTools, University of Freiburg, 79110, Freiburg, Germany, ${ }^{2}$ Faculty of Biology, University of Freiburg, 79104, Freiburg, Germany, \\ ${ }^{3}$ Computational Biology, School of Computer Science and Communication, KTH Royal Institute of Technology, 11428 Stockholm, Sweden, ${ }^{4}$ Bernstein \\ Center Freiburg, University of Freiburg, 79104, Freiburg, Germany, ${ }^{5}$ Department of Neurology, University of Michigan, Ann Arbor, Michigan 48109, \\ ${ }^{6}$ Institut des Maladies Neurodegeneratives, Universite de Bordeaux, 33076, Bordeaux, France, ${ }^{7}$ Department of Neurology and Kavli Institute for \\ Fundamental Sciences, University of California, San Francisco, California 94158, and ${ }^{8}$ Department of Psychology, University of Sheffield, Cathederal Court, \\ Sheffield, S1 1HD, United Kingdom
}

Brief epochs of beta oscillations have been implicated in sensorimotor control in the basal ganglia of task-performing healthy animals. However, which neural processes underlie their generation and how they are affected by sensorimotor processing remains unclear. To determine the mechanisms underlying transient beta oscillations in the LFP, we combined computational modeling of the subthalamopallidal network for the generation of beta oscillations with realistic stimulation patterns derived from single-unit data recorded from different basal ganglia subregions in rats performing a cued choice task. In the recordings, we found distinct firing patterns in the striatum, globus pallidus, and subthalamic nucleus related to sensory and motor events during the behavioral task. Using these firing patterns to generate realistic inputs to our network model led to transient beta oscillations with the same time course as the rat LFP data. In addition, our model can account for further nonintuitive aspects of beta modulation, including beta phase resets after sensory cues and correlations with reaction time. Overall, our model can explain how the combination of temporally regulated sensory responses of the subthalamic nucleus, ramping activity of the subthalamic nucleus, and movement-related activity of the globus pallidus leads to transient beta oscillations during behavior.

Key words: basal ganglia; beta oscillations; subthalamo-pallidal network

Significance Statement

Transient beta oscillations emerge in the normal functioning cortico-basal ganglia loop during behavior. Here, we used a unique approach connecting a computational model closely with experimental data. In this way, we achieved a simulation environment for our model that mimics natural input patterns in awake, behaving animals. We demonstrate that a computational model for beta oscillations in Parkinson's disease (PD) can also account for complex patterns of transient beta oscillations in healthy animals. Therefore, we propose that transient beta oscillations in healthy animals share the same mechanism with pathological beta oscillations in PD. This important result connects functional and pathological roles of beta oscillations in the basal ganglia.

\section{Introduction}

Exaggerated cortico-basal ganglia oscillations in the beta band $(15-30 \mathrm{~Hz})$ are a common feature of Parkinson's disease (PD) (Brown et al., 2001; Levy et al., 2002; Hammond et al., 2007).

\footnotetext{
Received May 11, 2017; revised Aug. 23, 2017; accepted Sept. 10, 2017.

Author contributions: R.S. designed research; A.M. performed research; A.M., D.L., N.M., and R.S. contributed unpublished reagents/analytic tools; A.M. analyzed data; A.M., A.K., D.L., N.M., A.A., J.B., and R.S. wrote the paper.

This work was supported by Brain Links-BrainTools Cluster of Excellence funded by the German Research Foundation (DFG Grant EXC 1086) and the University of Sheffield. We also acknowledge support by the state of BadenWürttemberg through bwHPC and DFG Grant INST 39/963-1 FUGG. We thank Wei Wei, Alejandro Jimenez, Lars Hunger, and Mohammad Mohagheghi Nejad for useful comments and discussion.

The authors declare no competing financial interests.
}

However, beta oscillations are not always pathological. Brief epochs of beta oscillations have been implicated in sensorimotor control in the healthy basal ganglia (Courtemanche et al., 2003; Berke et al., 2004; Leventhal et al., 2012; Feingold et al., 2015). These studies suggest that temporally regulated transient beta oscillations are important for normal functioning of the motor system.

Correspondence should be addressed to Amin Mirzaei, Faculty of Biology, University of Freiburg, Hansastrasse 9a 79104 Freiburg, Germany. E-mail: amin.mirzaei@brainlinks-braintools.uni-freiburg.de. DOI:10.1523/JNEUROSCI.1289-17.2017

Copyright $\odot 2017$ the authors $\quad 0270-6474 / 17 / 3711220-13 \$ 15.00 / 0$ 
The origin of beta oscillations in the cortico-basal ganglia system remains unknown. However, interactions between subthalamic nucleus (STN) and globus pallidus externa (GPe) can generate beta oscillations, as has been shown in experimental (Bevan et al., 2002; Tachibana et al., 2011) and computational (Terman et al., 2002; Kumar et al., 2011, Pavlides et al., 2015; Wei et al., 2015) studies. Anatomically, STN and GPe are densely and reciprocally interconnected (Shink et al., 1996). STN cells excite neurons in GPe (Kitai and Kita, 1987), which in turn receive inhibitory input from GPe (Smith et al., 1990; Parent and Hazrati, 1995). Such recurrent excitation-inhibition can generate oscillations (Plenz and Kital, 1999; Brunel, 2000), which may then propagate to other regions in the cortico-basal ganglia loop.

Beta oscillations have been proposed to play a functional role in maintaining the status quo in the motor system (Gilbertson et al., 2005; Engel and Fries, 2010). This idea has been supported by increased cortical beta-band activity during maintenance of a static position (Baker et al., 1997), active suppression of movement initiation (Swann et al., 2009), and postmovement hold periods (Pfurtscheller et al., 1996). Accordingly, beta power decreases in the cortico-basal ganglia loop during movement preparation and execution (Pfurtscheller et al., 2003; Sochurkova and Rektor, 2003; Kühn et al., 2004; Alegre et al., 2005). However, recent studies have indicated a more complex picture in which beta oscillations affect behavior through motor adaptation (Tan et al., 2014) and modulation of task performance (Feingold et al., 2015).

Supporting a more complex picture of beta oscillations, we provided evidence that basal ganglia beta oscillations are involved in sensorimotor processing and the utilization of cues for behavior (Leventhal et al., 2012). In particular, we found that beta power increases after sensory cues and movement initiation depended on how fast the animals reacted to a sensory cue. For short reaction times, LFP beta emerged after movement initiation, whereas for long reaction times, two separate beta epochs occurred, one before and one after movement initiation. In addition to modulation of beta power, we also observed that beta phases were affected by task events differently. Sensory cues, but not movement initiation, led to a short-latency phase reset in the beta band (Leventhal et al., 2012).

These complex oscillatory dynamics present both a challenge and an opportunity for understanding underlying cortico-basal ganglia circuit mechanisms. Currently, it is unknown whether pathological beta oscillations in PD share the same mechanisms with transient beta oscillations in healthy animals. If this is the case, then computational models for beta oscillations should be able to account for the complex beta dynamics in both healthy and parkinsonian animals. Recent network models of beta oscillations in PD have emphasized that, in addition to structural changes (e.g., connection strengths), changes in spiking activity of external inputs can promote beta oscillations (Kumar et al., 2011), which might drive transient beta oscillations. Here, we exploit this property by using activity patterns recorded in healthy rats during task performance (Schmidt et al., 2013; Mallet et al., 2016) as input to our computational model to study the resulting impact on the beta dynamics. Using this novel approach, we find that our model can account for the complex beta dynamics in the healthy rat LFP. Our results support overlapping mechanisms for pathological and healthy beta oscillations and provide the basis for studying the functional role of beta oscillations in network models.
Table 1. Comparison of model parameters in Kumar et al. (2011) and the modified model without recurrent STN connections

\begin{tabular}{ll}
\hline Kumar et al. (2011) & Modified model \\
\hline$C_{\text {STN-STN }}=0.02$ & $C P_{\text {STN-STN }}=0$ \\
$C P_{\text {STN-GPe }}=0.02$ & $C P_{\text {STN-GPe }}=0.022$ \\
$C P_{\text {GPe-STN }}=0.02$ & $C P_{\text {GPe-STN }}=0.035$ \\
$C P_{\text {GPe-GPe }}=0.02$ & $C P_{\text {GPe-GPe }}=0.02$ \\
$J_{\text {STN-STN }}=1.2$ & $J_{\text {STN-STN }}=-$ \\
$J_{\text {STN-GPe }}=1.2$ & $J_{\text {STN-GPe }}=1.2$ \\
$J_{\text {GPe-STN }}=-1.135$ & $J_{\text {GPe-STN }}=-0.8$ \\
$J_{\text {GPe-GPe }}=-0.725$ & $J_{\text {GPe-GPe }}=-0.725$ \\
$d_{\text {STN-STN }}=2$ & $d_{\text {STN-STN }}=-$ \\
$d_{\text {STN-GPe }}=5$ & $d_{\text {STN-GPe }}=6$ \\
$d_{\text {GPe-STN }}=5$ & $d_{\text {GPe-STN }}=6$ \\
$d_{\text {GPe-GPe }}=2$ & $d_{\text {GPe-GPe }}=3$ \\
\hline$P_{\text {COT }}$ & \\
\hline
\end{tabular}

$(P$, , Connection probability; J, synaptic weight; $d$ : delay (in ms).

\section{Materials and Methods}

Network model. The basic model structure and the parameter settings are the same as in Kumar et al. (2011). Briefly, the model includes 1000 excitatory STN neurons and 2000 inhibitory GPe neurons. Neurons were implemented as leaky integrate-and-fire neurons. Synaptic input was modeled as transient exponential conductance changes. All model neurons receive uncorrelated Poisson spike trains as inputs so as to achieve previously reported baseline activities for STN $(15 \mathrm{~Hz})$ and for GPe (45 Hz) (Bergman et al., 1994; Raz et al., 2000;). All network simulations were written in Python using pyNN as an interface to the simulation environment NEST (Gewaltig and Diesmann, 2007). Analysis of the simulation results and the LFP and single-unit data were performed using MATLAB R2013b (version 8.2.0.701; The MathWorks).

For the model variant without recurrent connections in STN (see Fig. 8 ), we used slightly different parameters for the connection probabilities, synaptic weights, and transmission delays (Table 1). Furthermore, the background Poisson input to the model neurons was adjusted so that the neurons had a broader distribution of baseline firing rates that closer matched the firing rate distribution in the rat data (Schmidt et al., 2013; Mallet et al., 2016).

Experimental design and statistical analysis. We combined previously recorded datasets of tetrode recordings in different basal ganglia subregions of rats performing a stop-signal task (for details, see Leventhal et al., 2012; Schmidt et al., 2013; Mallet et al., 2016). To exclude potential multiunit activity from our recordings, we only included units with $<1 \%$ of interspike intervals shorter than $1 \mathrm{~ms}$ in our dataset. The combined dataset contained 226 STN units from overall 40 recording sessions in five different rats, 149 putative prototypical GPe units from 41 recording sessions in four different rats, and 326 putative medium spiny neurons (MSNs) from 97 recording sessions in nine different rats. Between two recording sessions, tetrodes were typically moved by at least $80 \mu \mathrm{m}$ and we therefore considered units recorded in different sessions as different units. Animals performed a stop-signal task, but we only analyzed the subset of correct Go trials in which the animal moved contralateral to the recording site.

To identify STN neurons responding to the Go cue instructing contralateral movement (Fig. $1 C, D$ ), we used a shuffle test to determine whether neural activity increased significantly within $150 \mathrm{~ms}$ after the Go cue. The time of each spike within $-500 \mathrm{~ms}$ to $+200 \mathrm{~ms}$ relative to the Go cue was changed to a random spike time within the same time window. We then compared the number of actual spikes with the number of shuffled spikes in small time windows after the Go cue (15 nonoverlapping $10 \mathrm{~ms}$ windows $0-150 \mathrm{~ms}$ after the Go cue). We repeated this procedure 10,000 times and used the fraction of shuffles in which the number of shuffled spikes exceeded the number of actual spikes as the $p$-value to estimate statistical significance. STN neurons showing a $p$-value $<0.05 / 15$ for at least one bin after the time of the Go cue were considered sensory responsive. We performed the same shuffling method on GPe neurons to select movement-responsive GPe neurons (Fig. $1 F$ ), using all spikes within $-1 \mathrm{~s}$ to $+1 \mathrm{~s}$ relative to movement onset to detect firing rate changes for $50 \mathrm{~ms}$ time windows from $0-250 \mathrm{~ms}$ after 
movement onset (i.e., five nonoverlapping time bins). GPe neurons showing a $p$-value $<0.05 / 5$ for at least one bin after movement onset were considered movement responsive.

To identify movement-responsive MSNs in our single-unit data, average firing rates of MSNs were sorted based on their peak time within the interval from $1 \mathrm{~s}$ before to $1 \mathrm{~s}$ after movement initiation. MSNs with a peak firing rate between $150 \mathrm{~ms}$ before to $150 \mathrm{~ms}$ after movement onset were considered movement responsive MSNs $(n=100$; Fig. $1 E)$.

To determine whether a recorded unit showed a ramping firing pattern, we computed the average firing rates of each unit from one subregion over trials with a $50 \mathrm{~ms}$ sliding time window moving in steps of $10 \mathrm{~ms}$ from $1 \mathrm{~s}$ before the time of Go cue to the time of Go cue. Each resulting average firing rate was then normalized to values between 0 and 1 and then mean subtracted before applying principal component analysis. First, we computed the corresponding covariance matrix of all normalized zero-mean firing rates; then, we performed Eigen decomposition on the covariance matrix using the eig function of MATLAB. The projection $p$ of each normalized zero-mean average firing rate $r$ to the first eigenvector (corresponding to the maximum eigenvalue) was then computed as the normalized dot product: $p_{i}=\left\langle r_{i}, v_{i}\right\rangle / \lambda_{1}$, where $i$ is the unit index and $v_{1}$ the eigenvector with the largest eigenvalue $\lambda_{1}$. This yielded one projection value $p_{\mathrm{i}}$ for each recorded unit. Because the first eigenvector had a positive ramp over time, positive and negative projection values corresponded to positive and negative activity ramps of a recorded unit over time, respectively. The SD of the projection distribution from a random covariance matrix is $1 / \sqrt{n}$ (Anderson, 2003), with $n$ being the number of units. We considered neurons with a projection larger than $2 / \sqrt{n}$ or smaller than $-2 / \sqrt{n}$ as positive and negative ramp neurons, respectively (Fig. $2 A, B$ ). This analysis method was applied to determine positive and negative ramps in GPe and STN.

Modeling of sensory responses. To simulate sensory responses of STN neurons to the Go cue (Fig. $1 C, D$ ), we used inhomogeneous Poisson generators, each of which targeted one STN neuron in the model. The firing rate modulation of each inhomogeneous Poisson generator was a half-sine wave with a duration of $20 \mathrm{~ms}$ and maximum amplitude of $180 \mathrm{~Hz}$. The latency of the sensory stimulation for each STN neuron in the model was considered as the time interval between the peak of the half-sine wave and the time of the Go cue, which was taken randomly from the latency distribution of the sensory STN neurons in our experimental data (Fig. 1D). Because, in our single-unit data, $30 \%$ of the STN neurons responded to the Go cue, for each simulation, we targeted $30 \%$ of randomly chosen STN neurons as "sensory" STN neurons in the network model. Therefore, sensory responses in STN neurons could propagate in our network model to GPe, similar to some short latency responses we reported previously in GPe (Schmidt et al., 2013).

Modeling of motor responses. Firing rates of the movement-responsive MSNs (Fig. 1E) were summed up and used as the firing rate pattern of an inhomogeneous Poisson generator representing striato-pallidal movementrelated inhibition in the network model. Because $38 \%$ of the GPe neurons in our experimental data showed movement-related inhibition (Fig. $1 F)$, for each simulation, we targeted a randomly chosen $38 \%$ of the GPe neurons as "motor" GPe neurons in the network model.
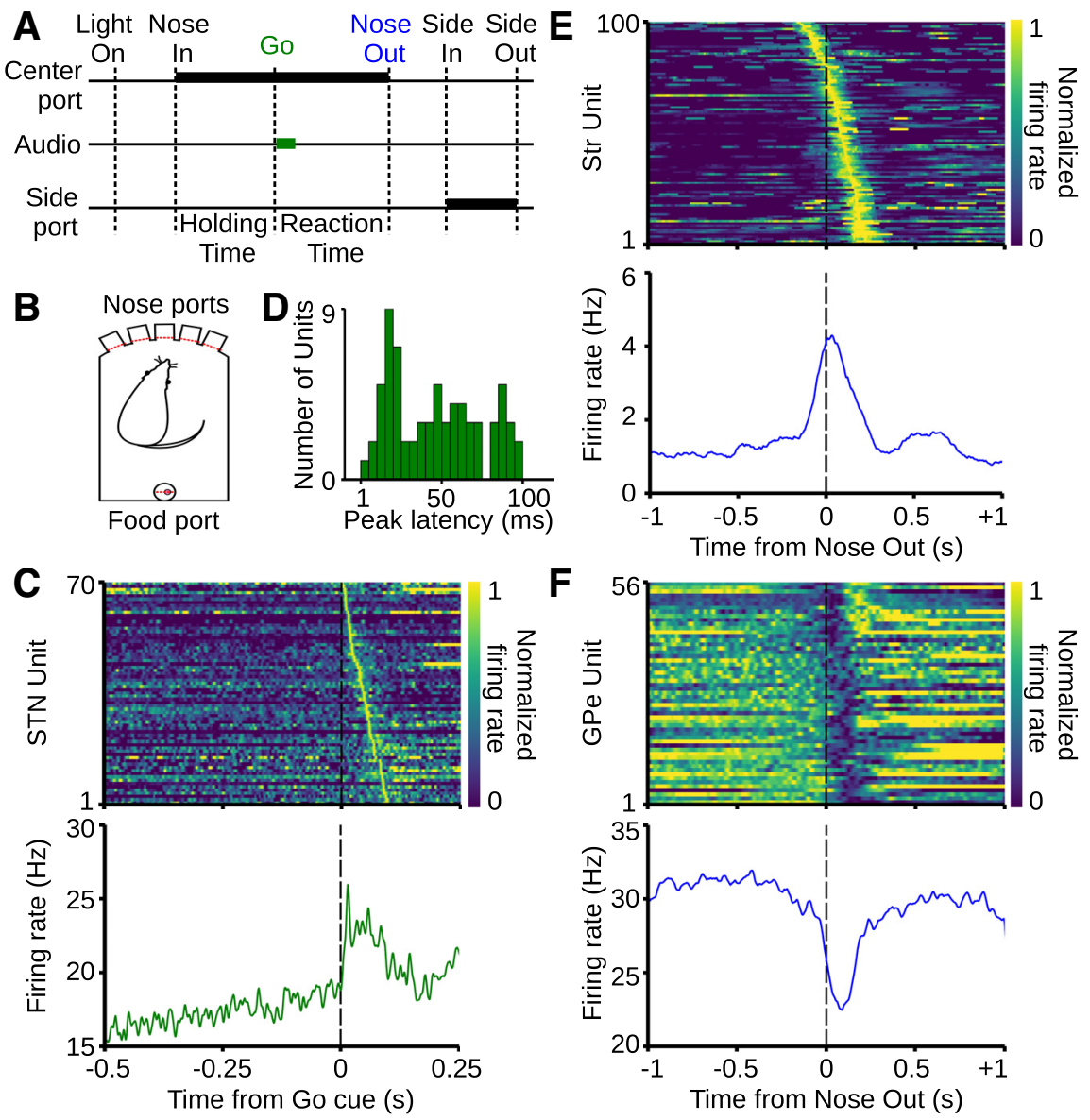

Figure 1. Single-unit responses to sensory and motor events during performance of the behavioral task. $\boldsymbol{A}$, Sequence of behavioral events during the experiment. Thick black bars show the position of the animal and thick green bar shows the occurrence of the sensory cue. Holding time refers to a random time delay (500-1200 ms) in which the animal waits in one of the three ports for the sensory cue. Reaction time is measured as the time between the onset of the Go cue and movement initiation activity of one unit). Bottom Corresponding mean firing rate of the STN subpopulation. D Distribution of peak latencies relative to the time of Go cue for STN neurons shown in $\boldsymbol{C}$. $\boldsymbol{E}$, Top, Normalized firing rates of single units in the striatum (putative MSNs) subpopulation. $\boldsymbol{F}$, Same as $\boldsymbol{E}$ but for the GPe subpopulation decreasing activity around movement onset

Modeling of firing rate ramps. To simulate the positive and negative ramps in the activity of the STN neurons observed before the Go cue (Fig. $2 A, B)$, for each simulation, we divided STN neurons in the network model into two nonoverlapping subpopulations. The fraction of STN neurons in each subpopulation in the network model was similar to the fraction we obtained from our experimental data (i.e., 34\% of neurons exhibited a positive ramp, $43 \%$ a negative ramp). We used an inhomogeneous Poisson generator with a positive ramp firing rate pattern as excitatory input to the positive ramp STN subpopulation in the model. The positive ramp in the firing rate of the inhomogeneous Poisson generator started $500 \mathrm{~ms}$ before the Go cue at $0 \mathrm{~Hz}$, reached $250 \mathrm{~Hz}$ at the time of the Go cue, and stayed constant until the movement onset (Fig. 3B). Such a stimulation lead to a $4 \mathrm{~Hz}$ increase in the activity of the positive ramp STN subpopulation in the network model during the $500 \mathrm{~ms}$ time interval preceding the Go cue, similar to what we observed in our experimental data (Fig. 2A).

Similarly, to simulate the negative ramp in the activity of STN neurons, we used another inhomogeneous Poisson generator with a positive ramp firing rate pattern as inhibitory input to the negative ramp STN model neuron subpopulation. The positive ramp in the firing rate of the inhibitory inhomogeneous Poisson generator started $500 \mathrm{~ms}$ before the time of Go cue at $0 \mathrm{~Hz}$, reached $350 \mathrm{~Hz}$ at the time of the Go cue, and stayed constant until the movement onset. Such a stimulation pattern lead to a 

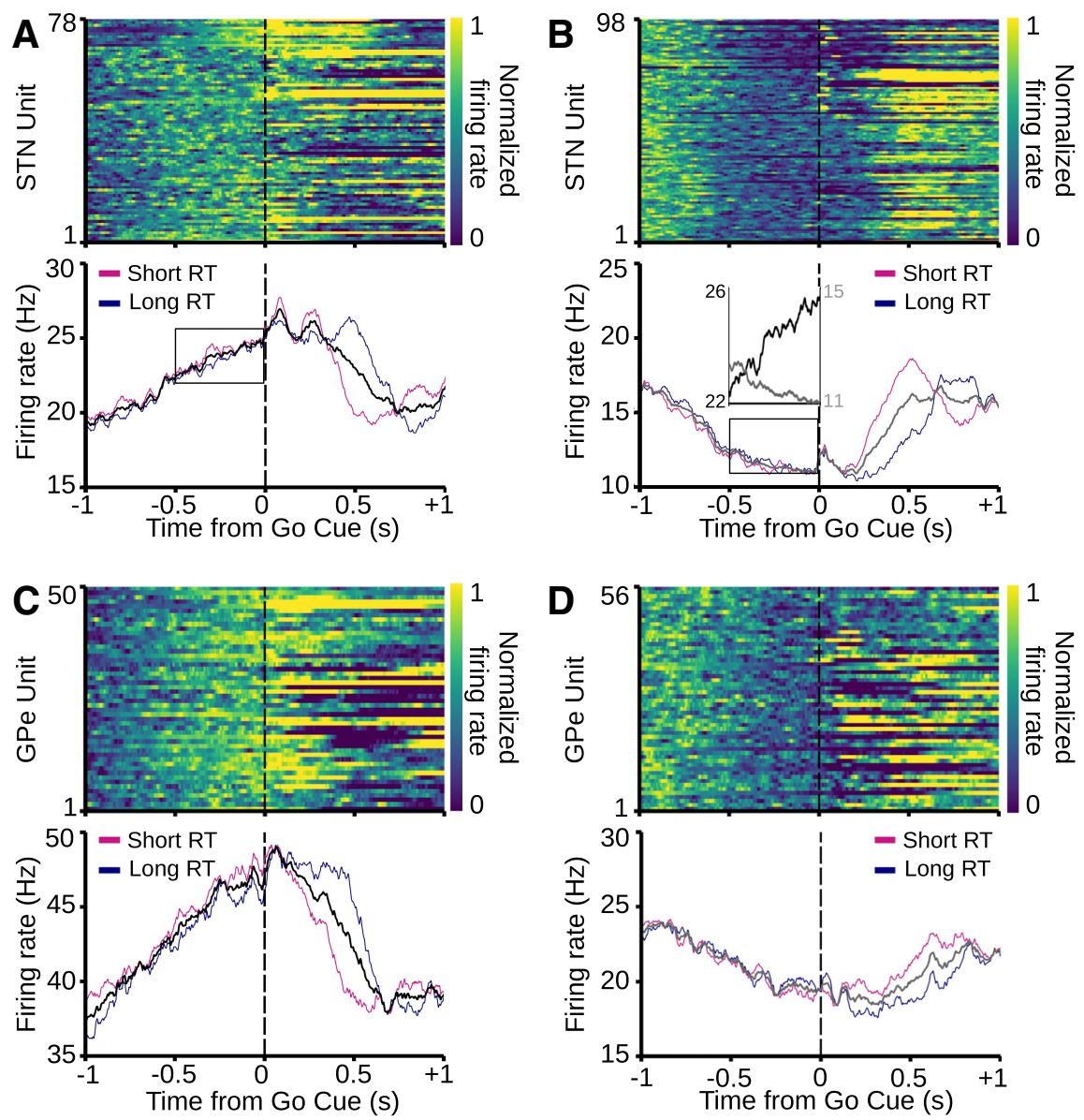

Figure 2. Ramping activity in STN and GPe while the animal is waiting for the Go cue. $\boldsymbol{A}$, Top, Normalized mean firing rate of single STN units with a positive ramp in firing rate before the Go cue. Bottom, Corresponding mean firing rate of the STN subpopulation in all trials (black) and subsets of long (cyan) and short (magenta) reaction time trials. $\boldsymbol{B}$, Same as $\boldsymbol{A}$ but for single STN units with a negative ramp in their firing rate before the Go cue. Inset, Direct comparison between average firing rates of neurons in $\boldsymbol{A}$ and $\boldsymbol{B}$ corresponding to the areas inside the black rectangles. $\boldsymbol{C}, \boldsymbol{D}$, Similar to $\boldsymbol{A}$ and $\boldsymbol{B}$, respectively, for GPe units.

\section{Results}

To determine whether a computational model for pathological beta oscillations in the STN-GPe network (Kumar et al., 2011) can account for complex beta dynamics during behavior in healthy animals, we devised realistic stimulation patterns for the network model based on single-unit recordings in rats performing a cued choice task (Schmidt et al., 2013; Mallet et al., 2016). At the beginning of each trial, the rat entered one of three center nose ports in an operant chamber ("Nose-in" event; Fig. $1 A, B$ ). The rat was trained to then hold its position for a variable time interval ("Holding time"; 500 $1200 \mathrm{~ms}$ ) until a Go cue instructed the rat to move its head quickly to the adjacent left or right side port ("Nose-out" event; Fig. $1 A, B)$. Correct performance of the task was rewarded with a sugar pellet. While the animals performed the task, we recorded GPe and STN in the striatum to determine activity patterns of single units during the time of the Go cue and during movement initiation. Then, we used these activity patterns to construct realistic input patterns for our network model. The network model that we use here is a large-scale spiking network model consisting STN and GPe populations with conductance-based synapses (Kumar et al., 2011; see Materials and Methods). Stimulating the network model via the realistic stimulation patterns allowed us to compare the resulting oscillatory dynamics in the model with properties of oscillations in the rat LFPs.
$1 \mathrm{~Hz}$ decrease in the activity of the negative ramp STN neurons in the network model during the $500 \mathrm{~ms}$ time interval preceding the Go cue, similar to what we observed in our experimental data (Fig. 2B).

Time-frequency analysis. The power spectrogram was computed by convolving $10 \mathrm{~s}$ of the GPe population firing rate (from -5 to $+5 \mathrm{~s}$ relative to the time of movement onset) in the model with a standard Morlet wavelet $(\sigma=0.849 / f)$ of integer frequencies $(f=1$ to $500 \mathrm{~Hz})$ and taking the logarithm of the squared magnitude of the resulting time series. To generate Figure $3 C$, bottom, we computed the mean spectrogram across 400 simulations of the model. The same method was used for GPe LFP data to generate Figure $3 C$, top. For each time point in the spectrogram, we summed the power in the beta range $(15-30 \mathrm{~Hz})$ and divided it by the summed power across all frequencies $(1-500 \mathrm{~Hz})$ to obtain continuous relative beta power, as shown in Figure 4, $A, B, E, F$, and Figure $6 B$.

Mean resultant length. The GPe population firing rate in the network model was convolved with the standard Morlet wavelet of each integer frequency in the beta band $(15-30 \mathrm{~Hz})$. For each frequency, the Hilbert transform of the filtered signal was computed to obtain a phase over time. The phase spread for each time point was then calculated by computing the length of the mean resultant vector over all trials using $M R L(t)$ $=\frac{1}{n} \sum_{n} e^{i \theta(n, t)}$, where $\theta(n, t)$ is the phase of the $n$th trial at time $t(n=400$ for the model). This results in a continuous measure of phase spread for each frequency in the beta range. The mean resultant lengths shown in Figure 4 were computed by taking the average across all beta frequencies.

\section{Brief, short-latency sensory responses in STN}

Thirty percent (70/226) of STN units responded to the Go cue with an increase in firing rate (Fig. $1 C$; shuffle test, $p<0.05 / 15$; see Materials and Methods). Consistent with our previous reports on a subset of the same data (Schmidt et al., 2013), this included units with a very short latency $(\sim 10-30 \mathrm{~ms})$ and responses of individual units were typically very brief (Fig. $1 C$, top). A potential source of such short-latency sensory responses of the STN units is the pedunculopontine tegmental nucleus (Pan and Hyland, 2005). In addition to the short-latency responses of the STN units, some STN units responded with a longer latency $(\sim 40-100 \mathrm{~ms})$, so that the overall distribution of peak response latencies had a bimodal shape (Fig. 1D). To mimic this STN response pattern to salient sensory stimuli, individual STN units received brief excitatory pulses with a fixed latency sampled from the latency distribution. These pulses were then used as input to 30\% randomly chosen STN model neurons (sensory STN neurons) to match the fraction of responding STN units in our single-unit data.

\section{Movement-related activity in striatum and GPe}

Thirty percent (100/320) of putative MSNs in the striatum increased their activity during contralateral movements (Fig. 1E; see Materials and Methods; also see Schmidt et al., 2013). We 
A
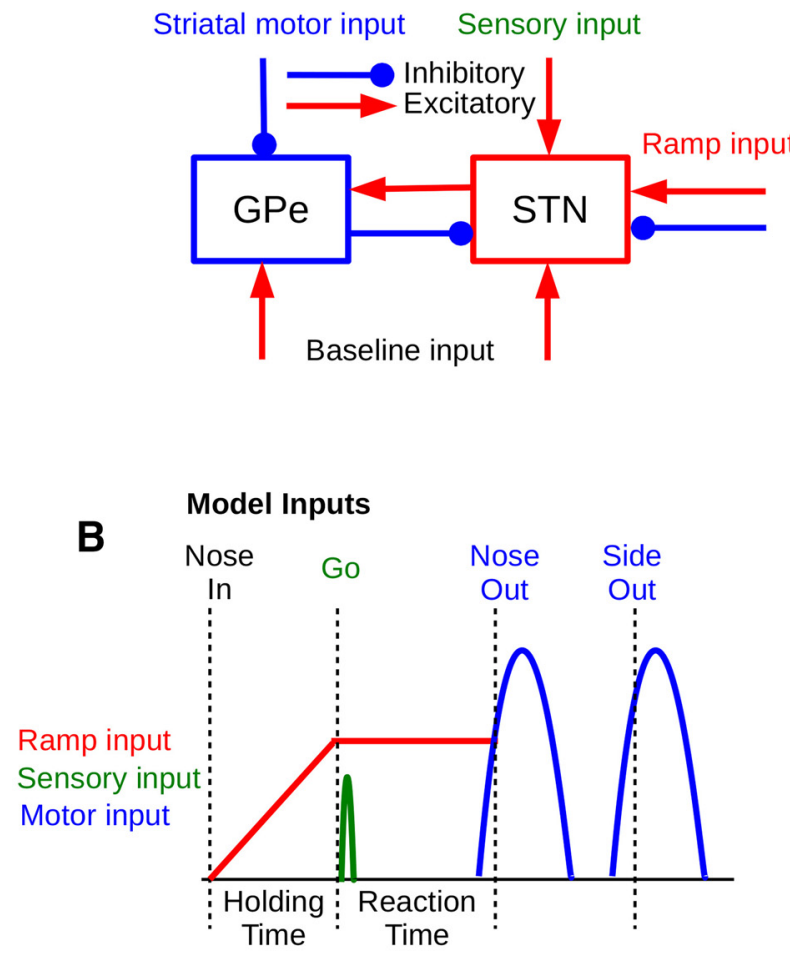
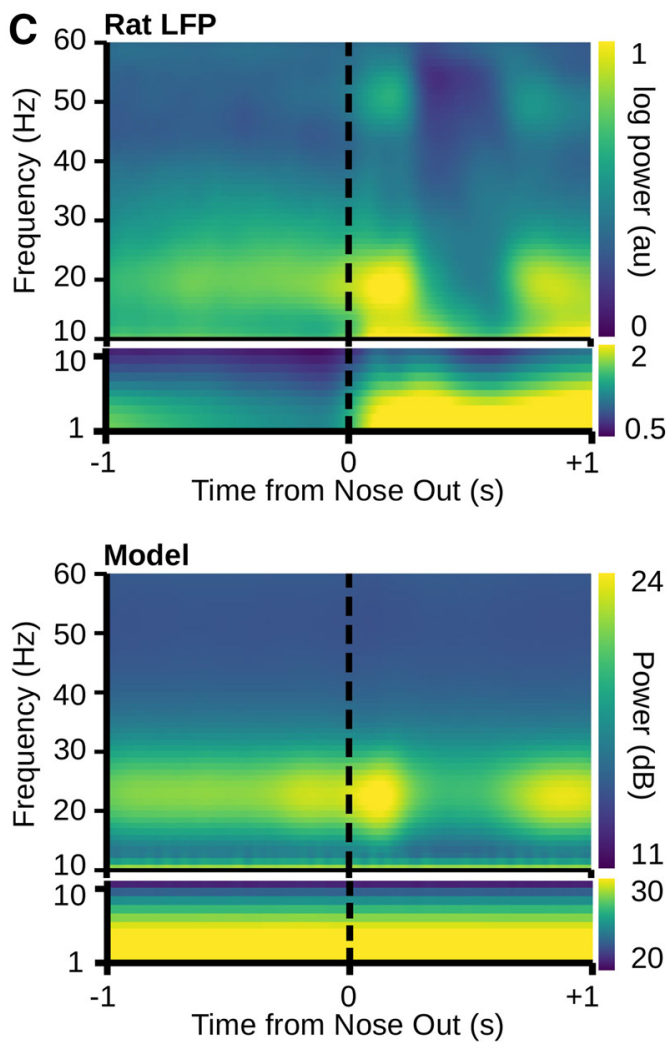

Figure 3. Computational model of beta oscillations stimulated with biologically realistic input patterns. $\boldsymbol{A}$, Scheme of the STN-GPe spiking neuronal network model. Motor input is provided as striatal inhibitory input to the GPe, whereas sensory input is provided as excitatory input to the STN. Ramp input comprises separate excitatory and inhibitory inputs to separate STN subpopulations (see Materials and Methods). B, Schematized temporal sequence of inputs to the network model during simulation of the behavioral task. $\boldsymbol{C}$, Top, Mean spectrogram of GPe LFP data showing modulation of LFP beta power during movement initiation. Bottom, Mean spectrogram (over 400 simulations) of GPe average firing rates for simulation of correct Go trials in the network model matching the time course of beta power in the experimental data.

focused here on contralateral movements because most neurons typically responded more during contralateral than ipsilateral movements (Gage et al., 2010; Schmidt et al., 2013). In GPe, 38\% $(56 / 149)$ of the units decreased their activity during contralateral movements (Fig. 1F; shuffle test, $p<0.05 / 5$; see Materials and Methods), possibly reflecting input from indirect pathway MSNs. Therefore, we assumed in the network model that striato-pallidal inhibition drives the GPe firing rate decreases during movement. We implemented this by generating inhomogeneous Poisson spike trains with a rate modulation following the MSN firing pattern during movement (Fig. $1 E$ ). These spike trains were then used as inhibitory inputs to $38 \%$ of the network model GPe neurons (motor GPe neurons) to match the fraction of GPe units with movement-related firing rate decreases in the single-unit data. Note that we restricted our analysis of GPe units to putative prototypical neurons (Mallet et al., 2016) because they receive input from MSNs and project to STN, whereas arkypallidal GPe neurons probably receive different inputs and do not project to STN (Mallet et al., 2016; Dodson et al., 2015).

\section{Ramping activity in STN and GPe while rats wait for the Go cue} In addition to single-unit responses that could be classified as sensory or motor, in STN and GPe, we found many units that exhibited a firing pattern that resembled a "ramp," a continuous change in firing rate. A ramping pattern was present in the activity of $77 \%$ $(176 / 226)$ of the STN units with either significantly increasing (positive ramp) or decreasing (negative ramp) firing rate while the animal was waiting for the Go cue (Fig. $2 A, B$ ). Among the 176 ramping STN units, $44 \%$ (78/176) showed positive ramps (Fig. $2 A)$ and $55 \%$
(98/176) showed negative ramps (Fig. 2B). However, the mean firing rate increase for the positive ramp units was four times as high as the mean firing rate decrease for the negative ramp units (4 vs $1 \mathrm{~Hz}$ decrease, respectively; Fig. $2 B$, bottom, inset). The positive ramp was also observed in the average firing rate of the whole STN population starting $500 \mathrm{~ms}$ before the Go cue (data not shown). Functionally, these ramps may correspond to a brake signal, preventing premature movement initiation (Frank, 2006).

We found a similar pattern in the GPe with 71\% (106/149) of the units exhibiting a significant ramping activity before the Go cue (Fig. $2 C, D$ ). Among these, $47 \%$ (50/106) showed positive ramps (Fig. 2C) and $52 \%$ showed negative ramps (Fig. $2 D$ ). Similar to the STN units, on average, the amplitude of the positive ramp in GPe was four times as high as the amplitude of the negative ramp, resulting in a net positive ramp in the population activity (data not shown). One property of the positive ramp STN and GPe units was that in long reaction time trials their activity remained elevated after the Go cue (Fig. 2A,C, bottom). This property played a key role for the beta dynamics in the model below.

Based on these ramping patterns in STN and GPe, we designed inputs to the model STN neurons that lead to similar activity ramps (see Materials and Methods). Due to the excitatory drive from STN to GPe, in the model, the ramps in STN activity resulted in corresponding ramps in GPe.

\section{Sensorimotor model inputs modulate time course of beta oscillations}

Because a previous modeling study demonstrated that excitatory input to STN or inhibitory input to GPe can induce transient beta 

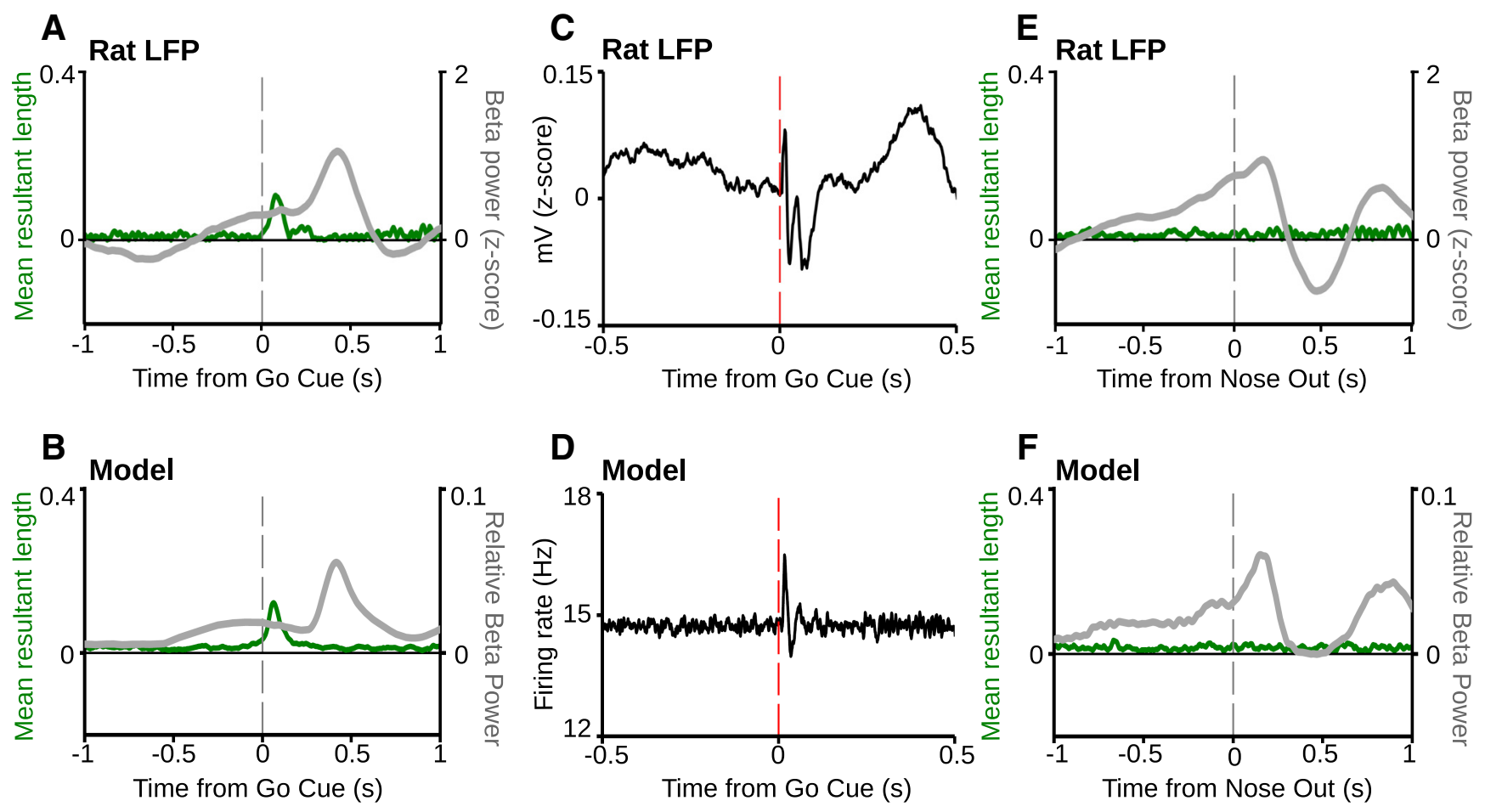

Figure 4. Sensory cues lead to a beta phase reset in both experimental data and in the network model. $\boldsymbol{A}, \boldsymbol{B}$, Time-resolved beta mean resultant length (left axes, green) and beta power (right axes, gray) of GPe LFP data during correctly performed contralateral go trials averaged across all rats ( $\boldsymbol{A}$ ) and of the network model GPe population firing rate (B) (average of 400 simulations). Note that sensory input is associated with a phase reset in both experimental data and in the model, shown as a brief increase in the value of the mean resultant length after the $G 0$ cue. $C$, Mean of the raw experimental STN LFP data over all correctly performed contralateral go trials aligned to the Go cue. $D$, Mean of the STN population firing rates in response to the Go cue in the network model (average of 400 simulations). $\boldsymbol{E}, \boldsymbol{F}$, Same analysis and simulations as in $\boldsymbol{A}$ and $\boldsymbol{B}$, respectively, but aligned to movement onset. Note that the phase distribution is random during initiation and execution of movement in both the rat and the network model (i.e., there was no increase in the mean resultant length around movement onset).

oscillations (Kumar et al., 2011), we hypothesized that the sequence of ramp, Go cue, and movement-related activity patterns (Fig. $3 A, B)$ accounts for the complex beta dynamics in the LFP (Leventhal et al., 2012). First, we reproduced the time course of beta power modulation during movement initiation (Leventhal et al., 2012) using an extended dataset of GPe recordings (Schmidt et al., 2013; Mallet et al., 2016). In the rat LFPs, beta power started to increase before the time of movement initiation and then showed a pronounced peak just after movement onset (Fig. 3C, top). The time course of beta power in the network model exposed to our singleunit stimulation patterns (Fig. $3 B$ ) matched the experimentally observed results (Fig. $3 C$, bottom), including the premovement betapower increase, the pronounced beta peak during movement, and the second beta peak related to the movement out of the side port (see Materials and Methods). The network model beta time course was in this case determined by the STN ramping activity, combined with the sensory responses of the STN neurons and the striatopallidal motor inputs (Fig. 3B). This is an important result because it connects single-unit activity during task performance with oscillatory network dynamics.

We compared the experimental LFP data with the model population firing rate (Fig. $3 C$ ). However, the origin of the LFP and its relation to spiking activity are not well understood in the basal ganglia. It seems that the LFP mostly reflects synchronized postsynaptic currents (Niedermeyer and Lopez da Silva, 1998; Jensen et al., 2005; Nunez and Srinivasan, 2005; McCarthy et al., 2011). However, we found that the time course of beta oscillations was very similar regardless of whether we used the population firing rate or the summation of IPSCs or EPSCs to represent the experimental LFP data (data not shown). Therefore, to stay consistent with previous models (Kumar et al., 2011; Nevado-Holgado et al., 2014;
Pavlides et al., 2015), we continue to use the population firing rate in the model to determine the presence of beta oscillations.

\section{Sensory responses in STN lead to a beta-phase reset}

In addition to the described changes in beta power, the phases of beta oscillations can be modulated by specific events in the behavioral task. Sensory cues (like the auditory Go cue) that did not lead to a distinctive increase in beta power were nevertheless followed by a short-latency phase reset in the LFP (Leventhal et al., 2012). In contrast, beta-power increases during movement were not accompanied by a phase reset in the beta band (Leventhal et al., 2012). We confirmed this result for GPe recording sites using an extended dataset (Schmidt et al., 2013; Mallet et al., 2016; Fig. $4 A, E$ ). To determine which properties of the neural signal lead to a phase reset or to a power increase in the beta band, we calculated grand averages of raw LFP traces (Fig. 4C). We found that, briefly after the Go cue, a single beta cycle was visible. This short oscillation was rather weak and could only be visible when looking at the mean of the LFP data over many trials (Fig. $4 C)$. This brief beta epoch was associated with beta phase reset in the LFP data after the Go cue (Fig. 4A). Interestingly, providing brief stimulation to the sensory STN neurons in the model leads to a brief, low-amplitude beta oscillation, which also only became visible when inspecting the mean population firing rate over many stimulations (Fig. 4D). Similar to the experimental data, sensory stimulation of the model STN leads to beta-phase reset in the ongoing activity of the network model (Fig. 4B). Therefore, we conclude that brief excitatory inputs to STN can induce weak, brief, phase-locked beta oscillations in the STN-GPe network, mimicking the experimentally observed results. 
Beta elevation around the time of movement onset was not accompanied by a phase reset in either the rat LFP data or in the model (Fig. 4E,F). It might seem counterintuitive that a strong stimulation leading to a clear increase in beta power did not reset the phase, whereas a weaker stimulation did. However, STN neuronal responses to the Go cue are brief compared with the longer movement-related increases in the activity of MSNs (Fig. $1 C-$ $E)$. Therefore, we hypothesized that the duration of neural responses to sensory and motor events might be the key difference. To test this, we varied the duration of the inputs to the model sensory STN neurons and motor GPe neurons systematically (note that the inputs are inhomogeneous Poisson spike trains with firing rate patterns of a half-cosine wave; see Materials and Methods). We found that, for brief inputs (leading to brief changes in the neuronal activity), there was a phase reset in the ongoing activity of the network model (Fig. 5). Longer stimulations of motor GPe neurons elevated the beta power without phase reset (Fig. 5C,D). For stimulation durations longer than a single beta period in the model (i.e., approximately $50 \mathrm{~ms}$ ), we only observed beta-power elevation without phase reset (Fig. $5 C, D$ ). In fact, the maximal phase reset in the network model occurred when the stimulation duration was $25 \mathrm{~ms}$, equaling half the beta cycle (Fig. $5 B, D)$. For the short stimulation duration, the time to get to the maximum of the half-cosine firing rate pattern is short (i.e., the slope is steeper). This effectively leads to no trial-to-trial variability because all realizations of the Poisson process with such a brief firing rate pattern are very similar (with respect to the spike times). This similarity in the input then leads to a similar response in the network model and therefore a phase reset across trials. In contrast, for longer stimulations, the time to get to the maximum of the half-cosine firing rate pattern is longer (with shallower slope). This leads to more trial-to-trial variability with respect to the spike times in the realization of the Poisson process. Correspondingly, this translates into trial-to-trial variability in the response of the network model to the long stimulation and therefore a random phase across trials.

Longer stimulations of the sensory STN neurons did not elevate the beta power in the network model (Fig. 5A). This is because sensory STN units made up a smaller fraction (30\%) of the STN population in our model compared with $38 \%$ motor GPe units (above). The long stimulation of a small fraction of the STN neurons was not sufficient to bring the network model into the oscillatory state. In general, for a certain stimulation strength, the fraction of stimulated neurons in the network model is a key parameter determining the amount of evoked beta power (Kumar et al., 2011).

\section{Disentangling the complex relationship between reaction time and beta dynamics}

The time course of beta oscillations depends on how fast the animal initiates movement in response to the Go cue (Leventhal et al., 2012). For short reaction times, the mean LFP beta power shows a single peak after movement initiation. For long reaction

\section{Inhibition of "motor" GPe neurons}
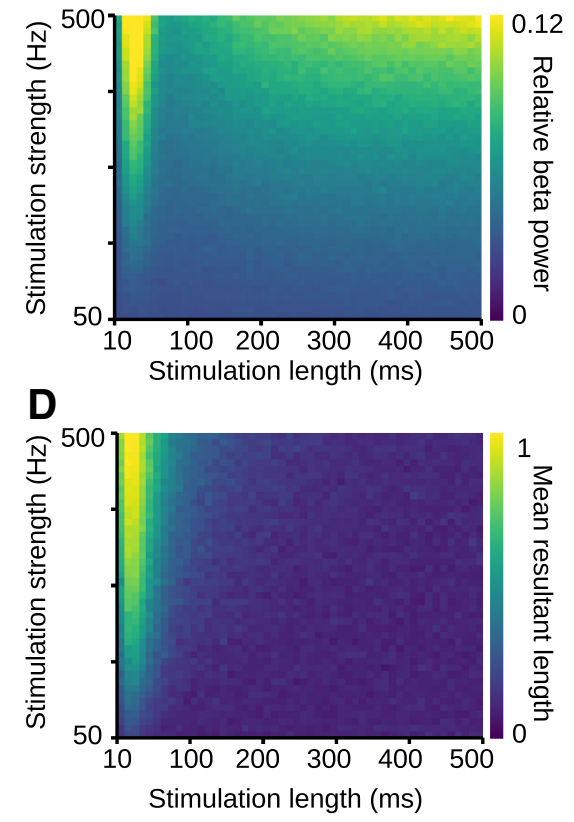

\section{.}

\section{2 咅}

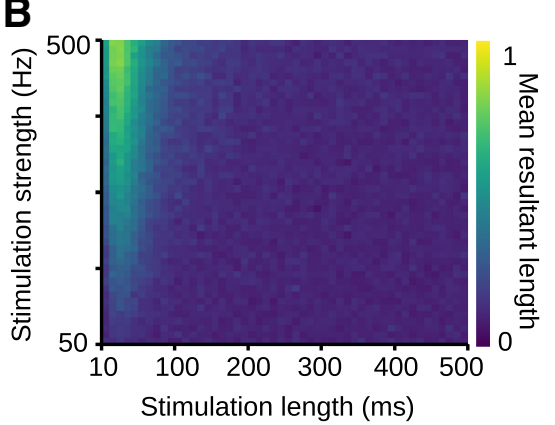

Figure 5. Effect of stimulation duration on beta power and phase reset in the network model. $\boldsymbol{A}, \boldsymbol{B}$, Relative beta power $(\boldsymbol{A})$ and caused by inhibitory input to the $38 \%$ motor GPe neurons (see Materials and Methods) of varying duration ( $x$-axis) and strength (y-axis). Note that, in all panels, we measured beta oscillations based on the GPe population firing rate.

times, the mean LFP beta power shows two peaks, the first before and the second after movement initiation (highlighted $300 \mathrm{~ms}$ epochs preceding and after "Nose-out" in Fig. 6A, right; see also Leventhal et al., 2012). The bimodal shape of the mean beta power for long reaction time trials is also visible when aligned to the Go cue (Fig. 6A, left). A straightforward idea would be that the first peak of the mean beta power for long reaction time trials is mostly driven by the Go cue or, alternatively, by the upcoming movement. However, if the beta peak were driven by the Go cue, then we would expect a higher peak for the data aligned to the Go cue than for the data aligned to movement onset. Accordingly, if the beta peak were related to the movement, then we would instead expect a higher peak for the data aligned to the movement onset. In contrast, despite variability in reaction time, this peak had a similar shape and amplitude for alignment to both the Go cue and movement onset. Therefore, this beta peak does not seem to be simply driven by a sensory or motor event. With the help of our network model, we disentangled the mechanisms underlying these reaction-time-dependent complex features of beta.

Using our stimulation patterns based on single-unit recordings, we studied how different reaction times affect the time course of beta power. We found a strikingly similar effect of reaction time on the time course of beta power in the network model (Fig. $6 B$ ). For long reaction time trials, the model exhibited two separate peaks in the mean beta power with the same time course as the experimental LFP data (Fig. 6B). Furthermore, the peak of the mean beta power in the model after movement onset for short reaction time trials had a higher amplitude than in long reaction time trials, similar to the experimental LFP data (Fig. 6A, $B$, right). The ability of the model to capture the fine details of the complex beta-power modulation became visible even at the single-trial level (Fig. 6C,D). As in the experimental data, changes in mean power modulation were reflected as a 
A

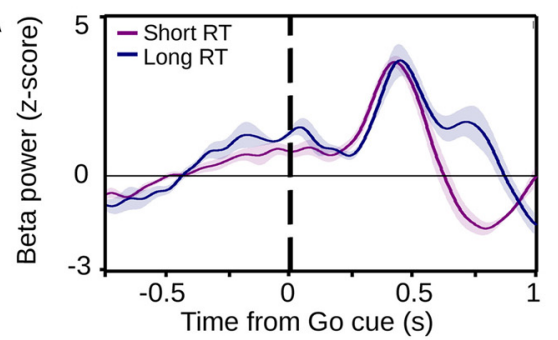

B

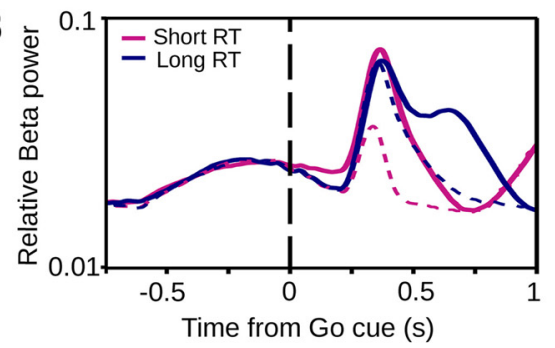

C

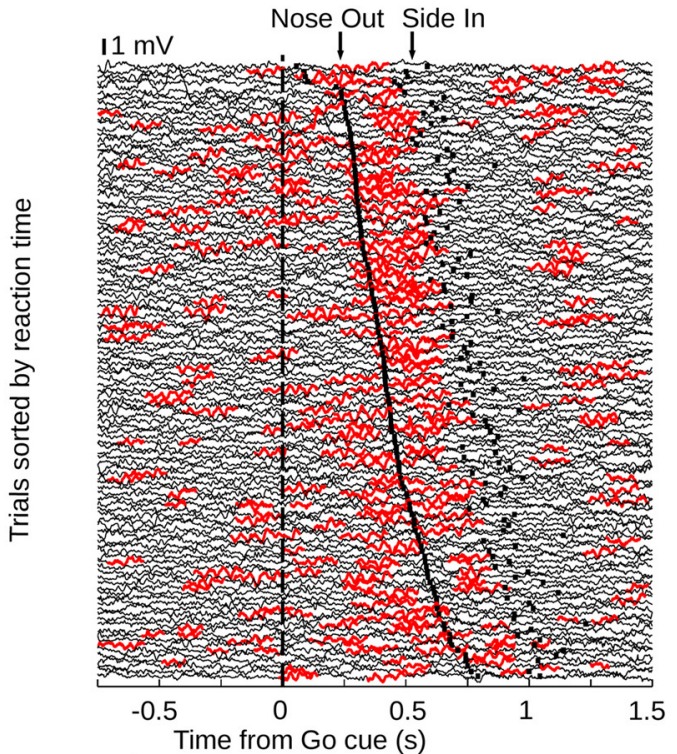

D

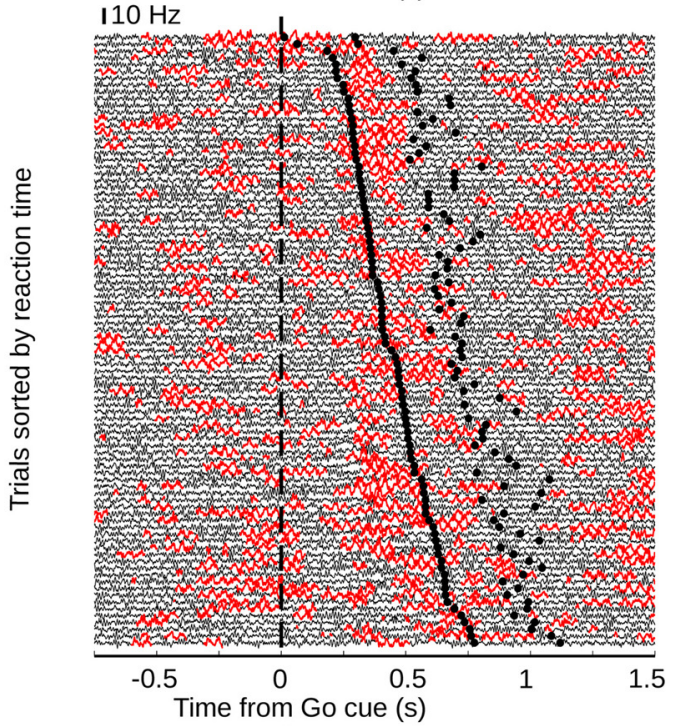

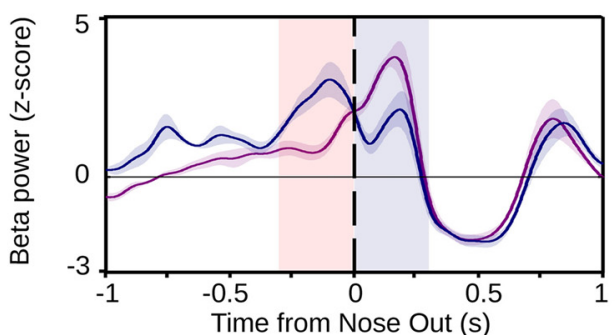
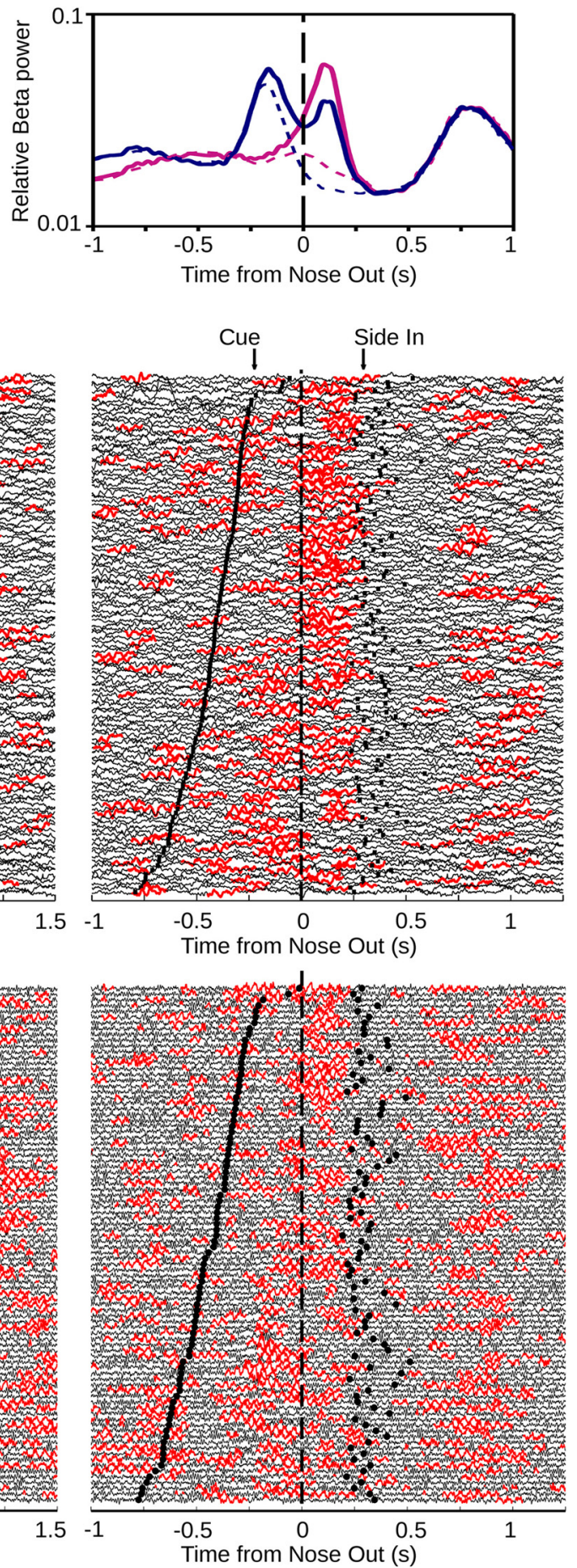

Figure 6. Relationship between beta oscillations and reaction time. $A$, Mean beta power of striatal LFP data for short ( $<500 \mathrm{~ms}$ ) and long ( $>500 \mathrm{~ms}$ ) reaction time trials aligned to the Go cue (left) and movement onset (right) averaged across rats (adapted with permission from Leventhal et al., 2012). B. Mean relative beta power of GPe population firing rates in the network model (averaged over 400 simulations) exposed to ramp, sensory, and motor stimulation patterns (solid lines). For comparison, if the striatal motor input to GPe is withheld in the model (dashed lines), then the second beta peak disappears for long reaction time trials (right, blue dashed line). C, Single-trial striatal LFP traces from a single recording session sorted by (Figure legend continues.) 
A

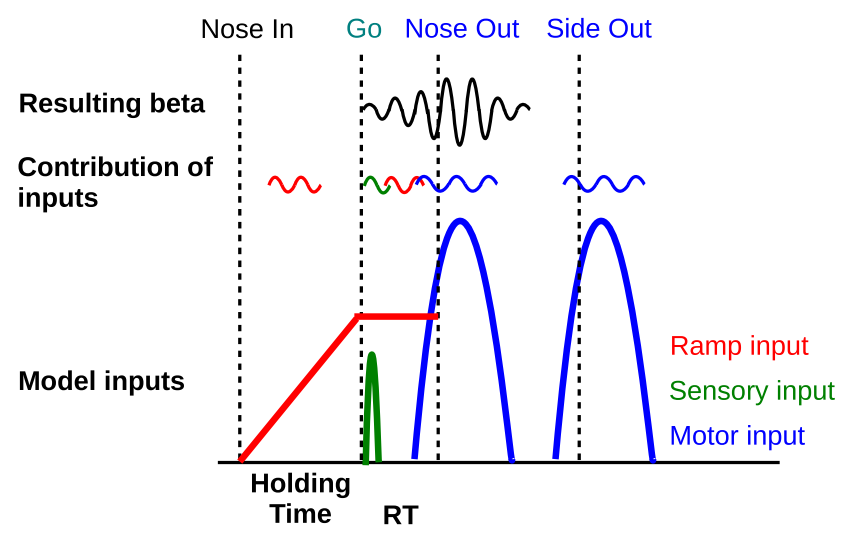

B

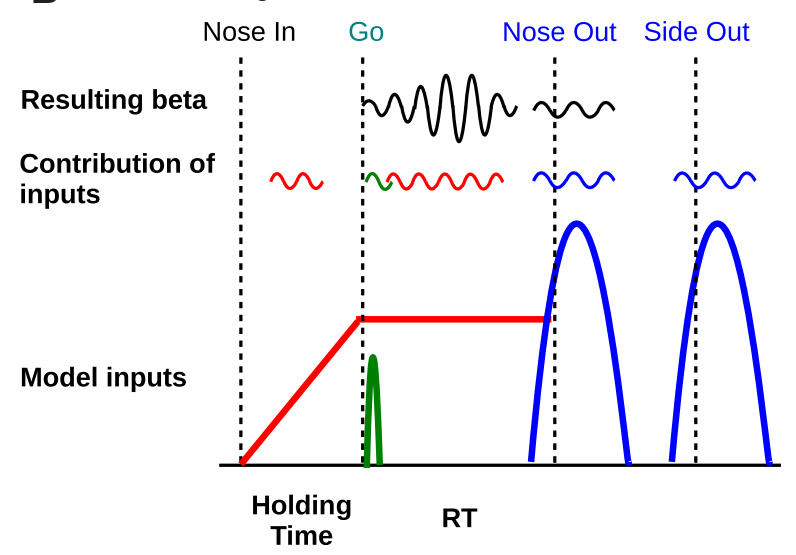

Figure 7. Scheme of contribution of each stimulation component to the generation of beta oscillations in short $(\boldsymbol{A})$, and long $(\boldsymbol{B})$ reaction time trials. Red, green, and blue schematized beta oscillations show the contribution of each individual input (ramp, sensory, and motor inputs, respectively) without the other one. Note that, for short reaction time trials, interaction between beta oscillations due to ramp, sensory, and motor inputs leads to transient increase in beta power around the time of movement onset (black trace shows the net effect of the interaction). For long reaction time trials, interaction between beta oscillations due to sensory and ramp inputs leads to transient increase in beta power before the time of movement onset, which is followed by another beta epoch due to motor input (black traces show the net effect of the interaction).

change in the probability of a transient beta oscillation rather than as only a gradual increase in the oscillation amplitude.

To understand the mechanisms underlying the complex relationship between beta and reaction times, we can now use our network model to determine the contribution of each stimulation component. Before the Go cue, ramping activity of the STN neurons in the model causes a gradual increase in beta power (mostly because of an increase in the probability of a beta event), starting almost $600 \mathrm{~ms}$ before the Go cue (Figs. $6 B, D, 7$ ). At the time of the Go cue, the sensory responses of the STN neurons generate a weak and brief beta oscillation in the model (Fig. 7, green traces). In short reaction time trials, this brief beta oscillation overlaps with beta oscillations driven by "ramp" and motor

$\leftarrow$

(Figure legend continued.) reaction time and aligned to the Go cue (left) and movement onset (right) with beta epochs marked in red (adapted with permission from Leventhal et al., 2012). D, Same visualization for single-trial model simulations with each trace showing the population firing rate of GPe neurons in the network model. For simulation of each trial, the model reaction time was selected randomly from the experimental data. inputs (because sensory and motor events are temporally close). This overlap results in an interaction of ongoing beta (driven by ramp input) with beta driven by motor input, leading to high beta power around the time of movement onset (Figs. 6B, 7, top). For long reaction time trials, after the Go cue but before movement initiation, the sensory and ramp inputs determine the beta dynamics in the model. The interaction between the sensory and ramp inputs leads to the first, high-amplitude beta peak for long reaction time trials (Figs. $6 B, 7$, bottom). Because Go cue and Nose-out events are temporally distant for long trials, this highamplitude beta power starts to decay before the time of movement onset. This is followed by another beta epoch due to motor input, which leads to the second peak of beta power after the time of movement onset for long reaction time trials (Figs. 6B, $D, 7$ ). The amplitude of this second peak is smaller compared with the peak after movement onset for short reaction time trials (Fig. $6 B$, right) because it lacks the interaction with STN excitation due to the Go cue (Fig. 7). Functionally, the first beta peak in long reaction time trials may be linked to the prolongation of movement initiation in high-beta states (Brown et al., 2001; Levy et al., 2002; Chen et al., 2007; Pogosyan et al., 2009). Therefore, our model connects ramp activity in STN with the generation of beta oscillations and potential functional roles as a "brake" (Frank, 2006).

Our results are robust to the STN-STN recurrent connectivity in the network model

In the network model that we used, the STN neurons receive excitatory synaptic inputs from other STN neurons with a connection probability of $2 \%$ (Kumar et al., 2011). However, several experimental studies indicated that the STN-STN recurrent connectivity is very rare or does not exist (Hammond and Yelnik, 1983; Sato et al., 2000; Parent and Parent, 2007; Koshimizu et al., 2013). Therefore, we modified the network model parameters to determine whether the model without STN-STN connections is also able to capture the behaviorally relevant dynamics of the LFP beta oscillations. Indeed, with slight modifications of parameters (see Materials and Methods), all key results, including the time course of beta around the time of movement preparation and execution (Fig. $8 A$ ), the beta phase reset (Fig. $8 B, C)$ and the complex relationship between beta and reaction time (Fig. $8 D, E$ ), were reproduced. This demonstrates that our model account of transient beta oscillations does not depend on STN-STN recurrent connectivity.

In summary, our results show that the combination of sensory responses of STN neurons, movement-related inhibition of GPe neurons, and ramping activity in STN account for the complex properties of beta-power modulation over time, beta-phase reset, and correlations with reaction time of rat electrophysiological recordings in the basal ganglia. Therefore, the model allows us to make clear predictions about the underlying mechanisms and provides the basis for studying functional consequences on neural processing and behavior.

\section{Discussion}

Oscillations in the LFP often reflect sensory, cognitive, and motor aspects of neural processing, but we lack understanding of how and why network oscillations emerge. Furthermore, we face a gap between firing patterns of single neurons and network dynamics. Here, we addressed this by combining experimental data with computational modeling to study how firing patterns in single units of task-performing healthy rats affect basal ganglia network dynamics. Although our computational model was originally used to describe beta oscillations in PD, this model also ac- 

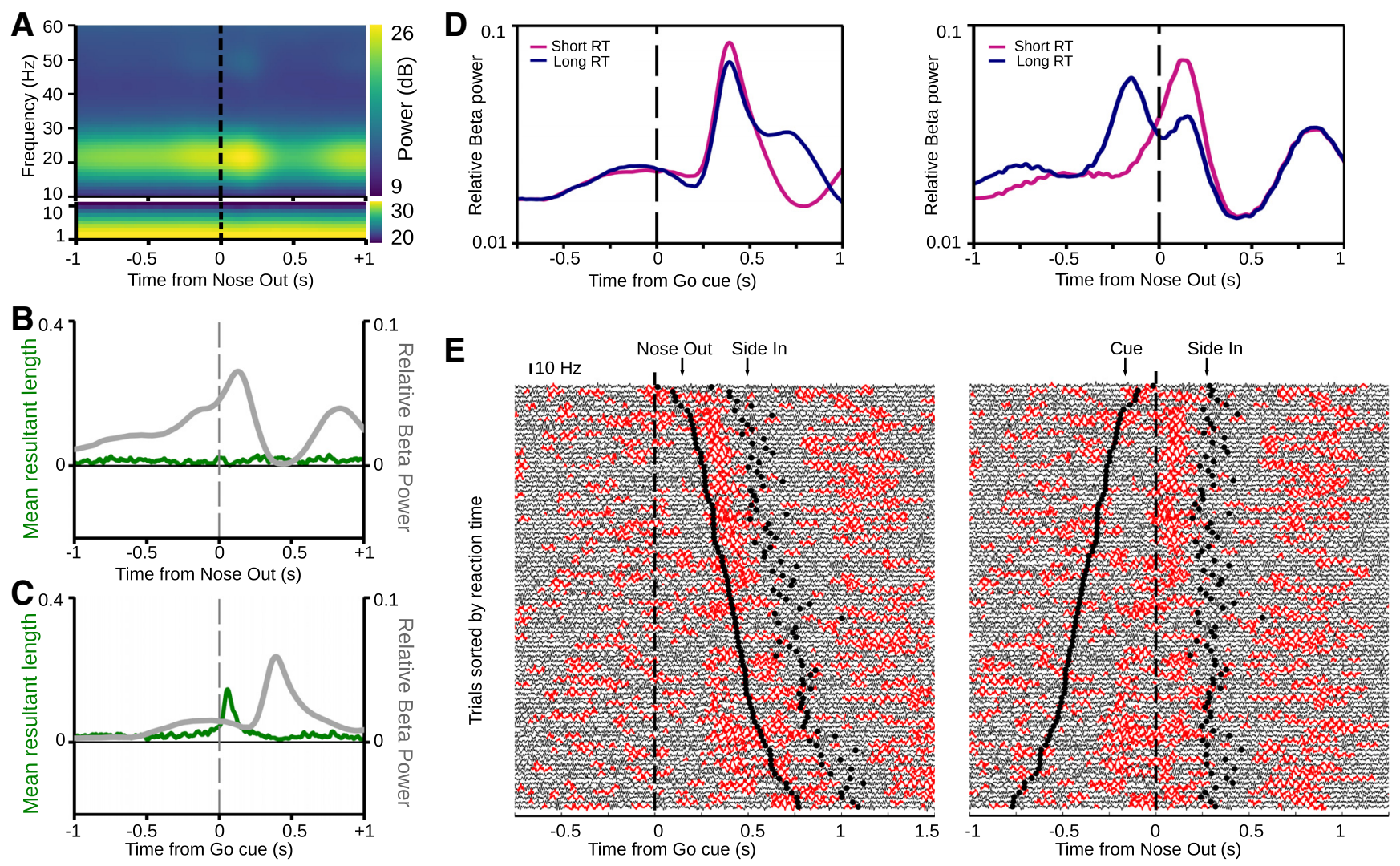

Figure 8. Network model without recurrent connections in STN reproduces all key results. $\boldsymbol{A}$, Mean spectrogram (over 400 simulations) of GPe average firing rates for simulation of correct Go trials in the modified network model matching the time course of beta power in the experimental data. $\boldsymbol{B}, \boldsymbol{C}$, Time-resolved beta mean resultant length (left axes, green) and beta power (right axes, gray) of the GPe population firing rate in the modified network model aligned to the movement onset ( $\boldsymbol{B})$ and to the Go cue ( $\boldsymbol{C}$; average of 400 simulations). $\boldsymbol{D}$, Mean relative beta power of GPe population firing rates in the modified network model aligned to the Go cue (left) and movement onset (right) averaged across 400 simulations. $\boldsymbol{E}$, Single-trial simulations of the modified network model sorted by reaction time, with each trace showing the population firing rate of GPe neurons aligned to the Go cue (left) and movement onset (right; beta epochs are marked in red).

counted for properties of beta in healthy animals. We characterize potential neuronal mechanisms underlying oscillations, relate healthy to pathological beta oscillations, and provide avenues for studying functional roles of beta in behavior.

\section{Neuronal mechanisms of beta oscillations}

Computational and experimental studies have implicated the STN-GPe network in beta oscillations in PD (Brown et al., 2001; Magill et al., 2001; Bevan et al., 2002; Terman et al., 2002; Rubin and Terman, 2004; Brown and Williams, 2005; Mallet et al., 2008; Tachibana et al., 2011; Stein and Bar-Gad, 2013; Nevado-Holgado et al., 2014; Pavlides et al., 2015; Wei et al., 2015). Moreover, corticosubthalamic excitation and striato-pallidal inhibition can generate beta oscillations in network models of the subthalamo-pallidal loop (Gillies et al., 2002; Kumar et al., 2011; Nevado-Holgado et al., 2014; Pavlides et al., 2015; Wei et al., 2015; Ahn et al., 2016). Consistently, we show that temporally regulated subthalamic excitation and pallidal inhibition reproduces the dynamics of transient beta oscillations observed in the healthy basal ganglia during behavior. Therefore, the same network that is responsible for beta oscillations in PD may also be involved in the generation of healthy beta.

As an alternative to the STN-GPe network, striatal MSNs (McCarthy et al., 2011), feedback projections from GPe back to striatum (Corbit et al., 2016), or spread of cortical beta to STN may be involved in basal ganglia beta oscillations. However, our model supports the role of the STN-GPe network due to the close correspondence between single-unit activity and the resulting complex time course of beta oscillations. Whether other models for the generation of beta would be able to account for the complex time course and behavioral correlates of beta remains to be shown. Although increased striatal spiking increases beta oscillations in several models (Kumar et al., 2011; McCarthy et al., 2011; Corbit et al., 2016), our model emphasizes the role of excitatory inputs to STN for the transient dynamics of beta oscillations. Overall, because beta oscillations are a heterogeneous phenomenon (Szurhaj et al., 2003; Kilavik et al., 2012; Feingold et al., 2015), the cortical and subcortical circuit may contain several mechanisms for the generation of beta, for example, to permit long-range communication (Fries, 2005). Therefore, these models are not necessarily exclusive and a key future challenge will be to disentangle the different circuits and their interaction. Nonetheless, we have shown that the STN-GPe network is sufficient to explain many features of beta oscillations in awake, behaving animals.

\section{Direct and indirect pathway MSNs}

Activity of direct pathway MSNs (striato-nigral) promote actions, whereas indirect pathway MSNs (striato-pallidal) suppress actions (Albin et al., 1989; Alexander and Crutcher, 1990; Kravitz et al., 2010; Freeze et al., 2013; Roseberry et al., 2016). Here, we considered movement-related increases in MSN activity (Fig. 1E) as inhibitory input to the model GPe (Fig. $3 A, B)$ without knowing whether the recorded MSNs are part of the direct or indirect pathway. This assumption is supported by evidence that direct and indirect pathway MSNs are concomitantly active during movements (Cui et al., 2013; Isomura et al., 2013). Nevertheless, 
there might be important activity differences between direct and indirect pathway neurons coordinating behavior. Whether coactivation of indirect pathway MSNs during movement reflects the suppression of alternative actions (Hikosaka et al., 2006; Redgrave et al., 2010) or activates specific neural assemblies in motor cortex (Oldenburg and Sabatini, 2015) remains unclear. Furthermore, almost $60 \%$ of direct pathway MSNs possess collateral terminal fields in GPe (Cazorla et al., 2014). Therefore, during movements, GPe likely receives increased inhibitory input from striatal MSNs, as incorporated in the model.

\section{STN as a brake}

We found ramps in the activity of STN units while the animal was waiting for the Go cue. During this time, the animal has to prevent premature movements to receive the food reward. Building on "hold-your-horses" models of STN (Frank, 2006), these ramps might prevent or delay movements. Correspondingly, in our experimental data, the ramps reached a plateau after the Go cue, which was linked to the reaction time (i.e., the plateau persisted longer in trials with a long reaction time; Fig. $2 A$, bottom). Therefore, these ramps might modulate the readiness for movement initiation. However, we also observed (data not shown) that the population activity of the STN ramps typically did last until movement initiation, indicating that the offset of this STN ramp does not provide a motor command itself. Instead, high STN activity might ensure that only coordinated movement commands (potentially signaled by striatal output), but not premature movement impulses, lead to motor output.

Conceptually, our model provides an important link among putative hold-your-horses ramping activity in STN, beta oscillations, and reaction times. The ramping activity increased spiking activity of the STN neurons and, consequently, also led to more beta oscillations in the model (Kumar et al., 2011). This was key in accounting for the bimodal shape of the mean beta power for long reaction time trials (Fig. $6 B$ ).

The STN ramps might be due to cortical drive. For example, in the motor cortex of monkeys, ramping activity has been observed while the animals anticipated sensory cues and needed to prevent premature movements (Confais et al., 2012). Furthermore, other cortical areas including right inferior frontal cortex and the presupplemental motor area project to STN and have been implicated in motor suppression (Wessel and Aron, 2017). In general, cortico-subthalamic excitation has been proposed to be important for the generation of beta oscillations (Tachibana et al., 2011; Pavlides et al., 2015). Importantly, the STN ramps during the hold period increased the probability of transient beta in our model. This fits well with antikinetic aspects of beta (Brown and Williams, 2005) and with STN activity correlating with slowness of movement observed during the progression of PD (Bergman et al., 1994; Remple et al., 2011).

\section{Behavioral relevance and predictions}

Beta oscillations seem to comprise a heterogeneous phenomenon with potentially different functions and mechanisms depending on the brain region (Szurhaj et al., 2003; Kilavik et al., 2012; Feingold et al., 2015). Here, we extend this view by proposing that transient, nonpathological basal ganglia beta can be driven by two distinct inputs. First, beta oscillations were driven by excitatory inputs to STN, including the ramping activity that might be linked to preventing premature movements. Second, beta oscillations were also driven by striato-pallidal inhibition during movement. Therefore, our model provides an explanation for why beta in some cases can be "antikinetic" (Brown and Williams,
2005), but in other cases can also appear during movement (Leventhal et al., 2012). Whether and how these two modes of beta make different functional contributions, for example, by differential communication with other brain regions (Fries, 2005), is an open question.

Based on our model, we make several experimentally testable predictions. First, the two modes of beta generation, GPe inhibition and STN excitation, might have different signatures in LFP recordings. If the beta is generated by GPe inhibition, then the oscillation begins with a decrease in GPe activity. If beta is generated by STN excitation, then the beta oscillation begins with an increase in STN. Although we do not know yet how spiking in the STN and GPe relates to patterns in the LFP, these two modes could translate into different onset phases of beta. Therefore, we presume that transient beta oscillations could be classified based on their onset phase and that this is indicative of whether the oscillation was driven by input to GPe or STN. Despite practical challenges such as detecting the exact onset phases of beta in noisy LFPs, this might provide valuable insights into whether the two modes of beta generation have distinct behavioral correlates.

Second, our model makes specific predictions about the relation between activity of MSNs projecting to GPe and the timing of beta oscillations (McCarthy et al., 2011). In recordings of identified direct and indirect pathway MSNs, our model predicts that the activity of the D2 MSNs predicts the timing of beta more accurately than the activity of the D1 MSNs. One complicating factor is that this distinction does not apply to beta driven by cortical excitation of STN.

Another model prediction arises from our observation that the duration of excitatory inputs to STN determines whether a phase reset occurs in the LFP. Sensory neuronal responses (Fig. $1 C, D)$ are typically brief. We propose that sensory cues from other modalities have the same effect so that, for example, visual cues that lead to brief excitations of STN also lead to a phase reset in the LFP signal. Furthermore, in addition to sensory cues, brief optogenetic stimulation of STN might yield the same effect. Whether these cue-induced beta-phase resets play also a functional role, for example, in the temporal coordination with inputs from other regions remains to be shown.

Finally, we predict that changes in the structure of the STN ramping activity affects the probability of beta oscillations. If the STN ramps indeed reflect a hold-your-horses signal (Frank, 2006), then the changes in the behavioral paradigm that manipulate the readiness for movement initiation should affect the ramping activity directly. For example, if the cost for the animal of a premature response is increased, then the corresponding ramping activity might change its time course and amplitude. In the model, this would translate directly into changes in the time course and probability of transient beta.

In conclusion, the direct combination of our computational model with experimental data provides a connection between single-unit activity and network oscillations. This helps us to study the functional contributions of transient beta oscillations during sensorimotor processing in a behavioral context.

\section{References}

Ahn S, Zauber SE, Worth RM, Rubchinsky LL (2016) Synchronized betaband oscillations in a model of the globus pallidus-subthalamic nucleus network under external input. Front Comput Neurosci 10:134. CrossRef Medline

Albin RL, Young AB, Penney JB (1989) The functional anatomy of basal ganglia disorders. Trends Neurosci 12:366-375. CrossRef Medline

Alegre M, Alonso-Frech F, Rodríguez-Oroz MC, Guridi J, Zamarbide I, Valencia M, Manrique M, Obeso JA, Artieda J (2005) Movement-related 
changes in oscillatory activity in the human subthalamic nucleus: ipsilateral vs. contralateral movements. Eur J Neurosci 22:2315-2324. CrossRef Medline

Alexander GE, Crutcher MD (1990) Functional architecture of basal ganglia circuits: neural substrates of parallel processing. Trends Neurosci 13:266271. CrossRef Medline

Anderson TW (2003) An introduction to multivariate statistical analysis, Ed 3. New York: Wiley.

Baker SN, Olivier E, Lemon RN (1997) Coherent oscillations in monkey motor cortex and hand muscle EMG show task-dependent modulation. J Physiol 501:225-241. CrossRef Medline

Bergman H, Wichmann T, Karmon B, DeLong MR (1994) The primate subthalamic nucleus. II. Neuronal activity in the MPTP model of parkinsonism. J Neurophysiol 72:507-520. Medline

Berke JD, Okatan M, Skurski J, Eichenbaum HB (2004) Oscillatory entrainment of striatal neurons in freely moving rats. Neuron 43:883896. CrossRef Medline

Bevan MD, Magill PJ, Terman D, Bolam JP, Wilson CJ (2002) Move to the rhythm: oscillations in the subthalamic nucleus-external globus pallidus network. Trends Neurosci 25:525-531. CrossRef Medline

Brown P, Williams D (2005) Basal ganglia local field potential activity: character and functional significance in the human. Clin Neurophysiol 116: 2510-2519. CrossRef Medline

Brown P, Oliviero A, Mazzone P, Insola A, Tonali P, Di Lazzaro V (2001) Dopamine dependency of oscillations between subthalamic nucleus and pallidum in Parkinson's disease. J Neurosci 21:1033-1038. Medline

Brunel N (2000) Dynamics of sparsely connected networks of excitatory and inhibitory spiking neurons. J Comput Neurosci 8:183-208. CrossRef Medline

Cazorla M, de Carvalho FD, Chohan MO, Shegda M, Chuhma N, Rayport S, Ahmari SE, Moore H, Kellendonk C (2014) Dopamine D2 receptors regulate the anatomical and functional balance of basal ganglia circuitry. Neuron 81:153-164. CrossRef Medline

Chen CC, Litvak V, Gilbertson T, Kühn A, Lu CS, Lee ST, Tsai CH, Tisch S, Limousin P, Hariz M, Brown P (2007) Excessive synchronization of basal ganglia neurons at $20 \mathrm{~Hz}$ slows movement in Parkinsons disease. Exp Neurol 205:214-221. CrossRef Medline

Confais J, Kilavik BE, Ponce-Alvarez A, Riehle A (2012) On the anticipatory precue activity in motor cortex. J Neurosci 32:15359-15368. CrossRef Medline

Corbit VL, Whalen TC, Zitelli KT, Crilly SY, Rubin JE, Gittis AH (2016) Pallidostriatal projections promote $\beta$ oscillations in a dopamine-depleted biophysical network model. J Neurosci 36:5556-5571. CrossRef Medline

Courtemanche R, Fujii N, Graybiel AM (2003) Synchronous, focally modulated beta-band oscillations characterize local field potential activity in the striatum of awake, behaving monkeys. J Neurosci 23:11741-11752. Medline

Cui G, Jun SB, Jin X, Pham MD, Vogel SS, Lovinger DM, Costa RM (2013) Concurrent activation of striatal direct and indirect pathways during action initiation. Nature 494:238-242. CrossRef Medline

Dodson PD, Larvin JT, Duffell JM, Garas FN, Doig NM, Kessaris N, Duguid IC, Bogacz R, Butt SJ, Magill PJ (2015) Distinct developmental origins manifest in the specialized encoding of movement by adult neurons of the external globus pallidus. Neuron 86:501-513. CrossRef Medline

Engel AK, Fries P (2010) Beta-band oscillations: signalling the status quo? Curr Opin Neurobiol 20:156-165. CrossRef Medline

Feingold J, Gibson DJ, DePasquale B, Graybiel AM (2015) Bursts of beta oscillation differentiate postperformance activity in the striatum and motor cortex of monkeys performing movement tasks. Proc Natl Acad Sci U S A 112:13687-13692. CrossRef Medline

Frank MJ (2006) Hold your horses: a dynamic computational role for the subthalamic nucleus in decision making. Neural Netw 19:1120-1136. CrossRef Medline

Freeze BS, Kravitz AV, Hammack N, Berke JD, Kreitzer AC (2013) Control of basal ganglia output by direct and indirect pathway projection neurons. J Neurosci 33:18531-18539. CrossRef Medline

Fries P (2005) A mechanism for cognitive dynamics: neuronal communication through neuronal coherence. Trends Cogn Sci 9:474-480. CrossRef Medline

Gage GJ, Stoetzner CR, Wiltschko AB, Berke JD (2010) Selective activation of striatal fast-spiking interneurons during choice execution. Neuron 67: 466-479. CrossRef Medline
Gewaltig M-O, Diesmann M (2007) NEST (Neural Simulation Tool) Scholarpedia 2:1430. CrossRef

Gilbertson T, Lalo E, Doyle L, Di Lazzaro V, Cioni B, Brown P (2005) Existing motor state is favored at the expenses of new movement during $13-35 \mathrm{~Hz}$ oscillatory synchrony in the human corticospinal system. J Neurosci 25:7771-7779. CrossRef Medline

Gillies A, Willshaw D, LiZ (2002) Subthalamic-pallidal interactions are critical in determining normal and abnormal functioning of the basal ganglia. Proc Biol Sci 269:545-551. CrossRef Medline

Hammond C, Yelnik J (1983) Intracellular labelling of rat subthalamic neurones with horseradish peroxidase: computer analysis of dendrites and characterization of axon arborization. Neuroscience 8:781-790. CrossRef Medline

Hammond C, Bergman H, Brown P (2007) Pathological synchronization in Parkinson's disease: networks, models and treatments. Trends Neurosci 30:357-364. CrossRef Medline

Hikosaka O, Nakamura K, Nakamura H (2006) Basal ganglia orient eyes to reward. J Neurophysiol 95:567-584. Medline

Isomura Y, Takekawa T, Harukuni R, Handa T, Aizawa H, Takada M, Fukai T (2013) Reward-modulated motor information in identified striatum neurons. J Neurosci 33:10209-10220. Medline

Jensen O, Goel P, Kopell N, Pohja M, Hari R, Ermentrout B (2005) On the human sensorimotor-cortex beta rhythm: sources and modeling. Neuroimage 26:347-355. CrossRef Medline

Kilavik BE, Ponce-Alvarez A, Trachel R, Confais J, Takerkart S, Riehle A (2012) Context-related frequency modulations of macaque motor cortical LFP beta oscillations. Cereb Cortex 22:2148-2159. CrossRef Medline

Kitai ST, Kita H (1987) Anatomy and physiology of the subthalamic nucleus: a driving force of the basal ganglia. In: The basal ganglia II: structure and function; current concepts (Carpenter MB, Jayaraman A, eds), pp 357-373. New York: Plenum.

Koshimizu Y, Fujiyama F, Nakamura KC, Furuta T, Kaneko T (2013) Quantitative analysis of axon bouton distribution of subthalamic nucleus neurons in the rat by single neuron visualization with a viral vector. J Comp Neurol 521:2125-2146. CrossRef Medline

Kravitz AV, Freeze BS, Parker PR, Kay K, Thwin MT, Deisseroth K, Kreitzer AC (2010) Regulation of parkinsonian motor behaviours by optogenetic control of basal ganglia circuitry. Nature 466:622-626. CrossRef Medline

Kühn AA, Williams D, Kupsch A, Limousin P, Hariz M, Schneider GH, Yarrow K, Brown P (2004) Event-related beta desynchronization in human subthalamic nucleus correlates with motor performance. Brain 127: 735-746. CrossRef Medline

Kumar A, Cardanobile S, Rotter S, Aertsen A (2011) The role of inhibition in generation and controlling Parkinson's disease oscillations in the basal ganglia. Front Syst Neurosci 5:86. CrossRef Medline

Leventhal DK, Gage GJ, Schmidt R, Pettibone JR, Case AC, Berke JD (2012) Basal ganglia beta oscillations accompany cue utilization. Neuron 73:523536. CrossRef Medline

Levy R, Ashby P, Hutchison WD, Lang AE, Lozano AM, Dostrovsky JO (2002) Dependence of subthalamic nucleus oscillations on movement and dopamine in Parkinson's disease. Brain 125:1196-1209. CrossRef Medline

Magill PJ, Bolam JP, Bevan MD (2001) Dopamine regulates the impact of the cerebral cortex on the subthalamic nucleus-globus pallidus network. Neuroscience 106:313-330. CrossRef Medline

Mallet N, Pogosyan A, Márton LF, Bolam JP, Brown P, Magill PJ (2008) Parkinsonian beta oscillations in the external globus pallidus and their relationship with subthalamic nucleus activity. J Neurosci 28:1424514258. CrossRef Medline

Mallet N, Schmidt R, Leventhal D, Chen F, Amer N, Boraud T, Berke JD (2016) Arkypallidal cells send a stop signal to striatum. Neuron 89:308316. CrossRef Medline

McCarthy MM, Moore-Kochlacs C, Gu X, Boyden ES, Han X, Kopell N (2011) Striatal origin of the pathologic beta oscillations in Parkinson's disease. Proc Natl Acad Sci U S A 108:11620-11625. CrossRef Medline

Nevado-Holgado AJ, Mallet N, Magill PJ, Bogacz R (2014) Effective connectivity of the subthalamic nucleus-globus pallidus network during Parkinsonian oscillations. J Physiol 592:1429-1455. CrossRef Medline

Niedermeyer E, Lopes da Silva F (1998) Electroencephalography, Ed 4. Baltimore: Lippincott Williams \& Wilkins.

Nunez PL, Srinivasan R (2005) Electric fields of the brain. Oxford: OUP. 
Oldenburg IA, Sabatini BL (2015) Antagonistic but not symmetric regulation of primary motor cortex by basal ganglia direct and indirect pathways. Neuron 86:1174-1181. CrossRef Medline

Pan WX, Hyland BI (2005) Pedunculopontine tegmental nucleus controls conditioned responses of midbrain dopamine neurons in behaving rats. J Neurosci 25:4725-4732. CrossRef Medline

Parent A, Hazrati LN (1995) Functional anatomy of the basal ganglia. I. The cortico-basal ganglia-thalamo-cortical loop. Brain Res Rev 20:91-127. CrossRef Medline

Parent M, Parent A (2007) The microcircuitry of primate subthalamic nucleus. Parkinsonism Relat Disord 13:S292-S295. CrossRef Medline

Pavlides A, Hogan SJ, Bogacz R (2015) Computational models describing possible mechanisms for generation of excessive beta oscillations in Parkinson's disease. PLoS Comput Biol 11:e1004609. CrossRef Medline

Pfurtscheller G, Stancák A Jr, and Neuper C (1996) Post-movement beta synchronization: a correlate of an idling motor area? Electroencephalogr Clin Neurophysiol 98:281-293. CrossRef Medline

Pfurtscheller G, Graimann B, Huggins JE, Levine SP, Schuh LA (2003) Spatiotemporal patterns of beta desynchronization and gamma synchronization in corticographic data during self-paced movement. Clin Neurophysiol 114:1226-1236. CrossRef Medline

Plenz D, Kital ST (1999) A basal ganglia pacemaker formed by the subthalamic nucleus and external globus pallidus. Nature 400:677-682. CrossRef Medline

Pogosyan A, Gaynor LD, Eusebio A, Brown P (2009) Boosting cortical activity at Beta-band frequencies slows movement in humans. Curr Biol 19:1637-1641. CrossRef Medline

Raz A, Vaadia E, Bergman H (2000) Firing patterns and correlations of spontaneous discharge of pallidal neurons in the normal and the tremulous 1-methyl-4-phenyl- 1,2,3,6-tetrahydropyridine vervet model of parkinsonism. J Neurosci 20:8559-8571. Medline

Redgrave P, Rodriguez M, Smith Y, Rodriguez-Oroz MC, Lehericy S, Bergman H, Agid Y, DeLong MR, Obeso JA (2010) Goal-directed and habitual control in the basal ganglia: implications for Parkinson's disease. Nat Rev Neurosci 11:760-772. CrossRef Medline

Remple MS, Bradenham CH, Kao CC, Charles PD, Neimat JS, Konrad PE (2011) Subthalamic nucleus neuronal firing rate increases with Parkinson's disease progression. Mov Disord 26:1657-1662. CrossRef Medline

Roseberry TK, Lee AM, Lalive AL, Wilbrecht L, Bonci A, Kreitzer AC (2016) Cell-type-specific control of brainstem locomotor circuits by basal ganglia. Cell 164:526-537. CrossRef Medline

Rubin JE, Terman D (2004) High frequency stimulation of the subthalamic nucleus eliminates pathological thalamic rhythmicity in a computational model. J Comput Neurosci 16:211-235. CrossRef Medline
Sato F, Parent M, Levesque M, Parent A (2000) Axonal branching pattern of neurons of the subthalamic nucleus in primates. J Comp Neurol 424:142152. CrossRef Medline

Schmidt R, Leventhal DK, Mallet N, Chen F, Berke JD (2013) Canceling actions involves a race between basal ganglia pathways. Nat Neurosci 16:1118-1124. CrossRef Medline

Shink E, Bevan MD, Bolam JP, Smith Y (1996) The subthalamic nucleus and the external pallidum: two tightly interconnected structures that control the output of the basal ganglia in the monkey. Neuroscience 73:335-357. CrossRef Medline

Smith Y, Hazrati LN, Parent A (1990) Efferent projections of the subthalamic nucleus in the squirrel monkey as studied by the PHA-L anterograde tracing method. J Comp Neurol 294:306-323. CrossRef Medline

Sochurkova D, Rektor I (2003) Event-related desynchronization/synchronization in the putamen: an SEEG case study. Exp Brain Res 149:401-404. CrossRef Medline

Stein E, Bar-Gad I (2013) $\beta$ oscillations in the cortico-basal ganglia loop during parkinsonism. Exp Neurol 245:52-59. CrossRef Medline

Swann N, Tandon N, Canolty R, Ellmore TM, McEvoy LK, Dreyer S, DiSano M, Aron AR (2009) Intracranial EEG reveals a time- and frequencyspecific role for the right inferior frontal gyrus and primary motor cortex in stopping initiated responses. J Neurosci 29:12675-12685. CrossRef Medline

Szurhaj W, Derambure P, Labyt E, Cassim F, Bourriez JL, Isnard J, Guieu JD, Mauguière F (2003) Basic mechanisms of central rhythms reactivity to preparation and execution of a voluntary movement: a stereoelectroencephalographic study. Clin Neurophysiol 114:107-119. CrossRef Medline

Tachibana Y, Iwamuro H, Kita H, Takada M, Nambu A (2011) Subthalamopallidal interactions underlying parkinsonian neuronal oscillations in the primate basal ganglia. Eur J Neurosci 34:1470-1484. CrossRef Medline

Tan H, Jenkinson N, Brown P (2014) Dynamic neural correlates of motor error monitoring and adaptation during trial-to-trial learning. J Neurosci 34:5678-5688. CrossRef Medline

Terman D, Rubin JE, Yew AC, Wilson CJ (2002) Activity patterns in a model for the subthalamopallidal network of the basal ganglia. J Neurosci 22:2963-2976. Medline

Wei W, Rubin JE, Wang XJ (2015) Role of the indirect pathway of the basal ganglia in perceptual decision making. J Neurosci 35:4052-4064. CrossRef Medline

Wessel JR, Aron AR (2017) On the globality of motor suppression: unexpected events and their influence on behavior and cognition. Neuron 93:259-280. CrossRef Medline 